\title{
Can imaging impact the coronavirus pandemic?
}

\author{
Anirudh Kohli \\ Chief Radiologist Breach Candy Hospital Trust, 60 Bhulabhai Desai Road, Mumbai, Maharashtra, India. \\ E-mail: dranirudhkohli@gmail.com
}

In December 2019, an outbreak of severe acute respiratory syndrome (SARS COV-2) coronavirus infection occurred in Wuhan, Hubei province China. This spread across China and beyond. On February 12, 2020, WHO named the disease Coronavirus disease 2019 (COVID-19). With international air travel, the disease spread across the globe infecting nearly every country on the planet. On March 12, WHO notified COVID 19 as a pandemic in view of its global spread.

Previous Coronavirus epidemics SARS (2002), MERS (2012) and Influenza A subtype (H1N1) 2009, have demonstrated relatively similar and characteristic imaging findings. Subpleural patchy ground glass densities more so in the lung bases. [Figures 1 and 2]. With increasing severity of disease there is progression of ground glass densities to consolidation. [Figure 3] In severe cases there is diffuse alveolar damage - acute lung injury as well as complications of mechanical ventilation such as barotrauma [Figure 4]. Imaging findings in the current corona virus outbreak COVID-19 have demonstrated similar features on imaging. This is a great boon, as imaging specialists are already familiar with the appearances from SARS/H1N1 epidemics. In fact in the current pandemic it has been found characteristic HRCT

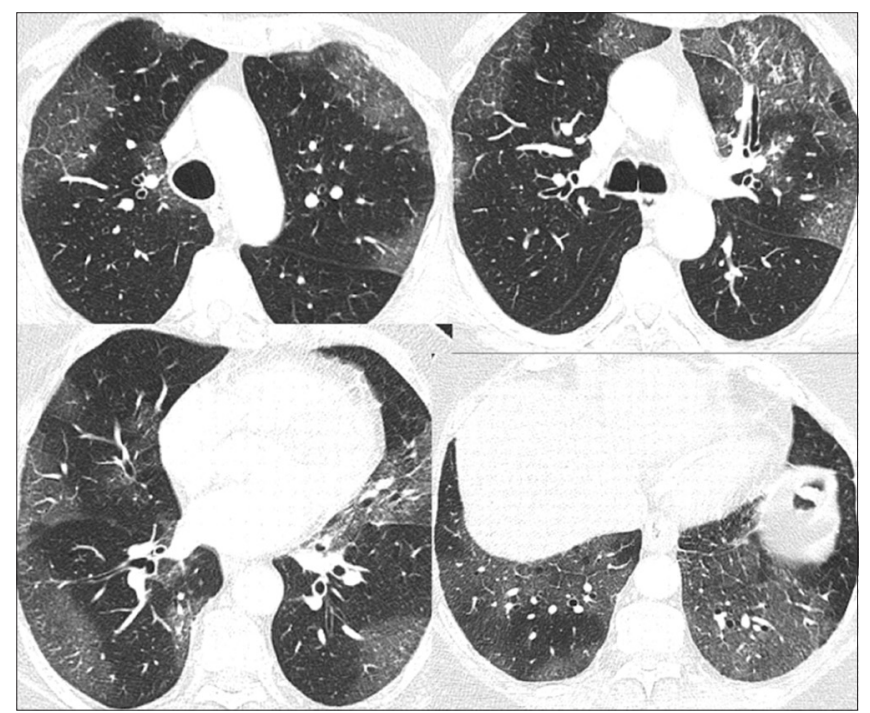

Figure 1: HRCT chest demonstrates bilateral sub pleural ground glass densities typical of viral pneumonia appearances appear at the onset of disease occasionally before real time reverse transcription polymerase chain reaction (RT_PCR) is positive which is used for definitive diagnosis of COVID-19. ${ }^{[1]}$ In fact a large study demonstrated HRCT to be positive in $88 \%$ of cases compared to RT PCR which was positive in $59 \%$ of cases..$^{[1]}$ In view of this significant high predictive value of HRCT and relative false negative rate of RT PCR, it has been aggressively suggested to utilise HRCT in surveillance for COVID-19. Considering the only means to control the pandemic is by identifying positive cases, isolating them and quarantining their contacts during incubation and infectious phases. South Korea, which was affected early in the pandemic, resorted to identifying every positive case, isolating and quarantine all contacts. They were very successful with first 30 cases. Case 31 was given a bit of leeway which resulted in $80 \%$ of south korea infections- from one individual. ${ }^{[2]}$ South Korea had more than 8000 infections. It is imperative to detect every possible case. In view of the importance to detect every case, the recommendation to use HRCT for surveillance may not be aggressive at all. In fact, may turn the tide in the pandemic. A head to head comparison between HRCT and RT PCR. HRCT is easily available across india and the world. There are more than $3000 \mathrm{CT}$ scanners in india. Nearly every district has one or multiple CT scanners. There are Only 52 RT PCR centres in india at this time. ${ }^{[3]}$ Time taken to obtain nasal swab and HRCT is relatively similar as just a breath hold scan required. Turn around time of result HRCT is

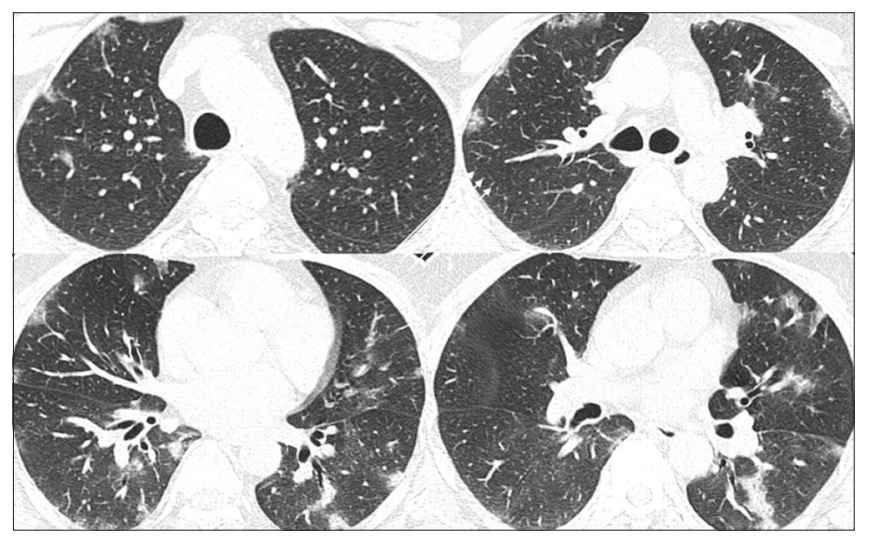

Figure 2: HRCT demonstrates subtle sub pleural densities in a case of viral pneumonia 


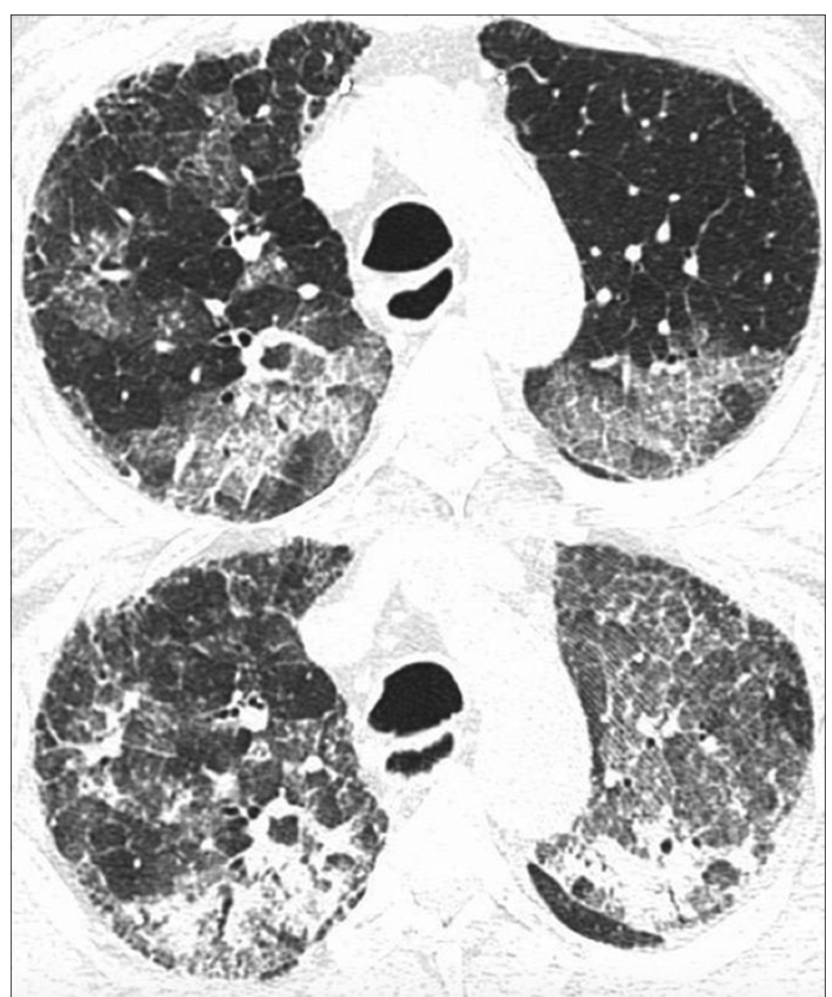

Figure 3: HRCT demonstrates ground glass densities in a sub pleural location (upper figure) which progress to consolidation as well as fresh ground glass densities (lower image) subsequently indicating disease worsening

instantaneous, RT PCR is 5 hours at present. Number of HRCT that can be done in a day could be 200 or much more per machine or more. Kasturba hospital mumbai has a capacity to do 300 RT PCR tests in a day. HRCT also has additional advantages as it helps assess the extent of pulmonary involvement, disease severity, thus helping to guide clinical management.

However ACR- American college of radiology has advised against utilisation of HRCT as a first line tool with a logic that RT PCR testing is the definitive diagnosis and will be required to establish the diagnosis. ${ }^{[3]}$

Management of a pandemic requires a multi pronged approach, innovative and unconventional methods. A lockdown is totally unconventional. HRCT could very well be used as an initial screening tool to fast track individuals in the RT PCR line which can be endless missing out positive cases. In view of the limited resources for RT PCR, this is essential, if every case needs to be detected.

Whether HRCT is utilised in surveillance or not, HRCT will be required during the pandemic to asses for disease severity and complications. Imaging specialists as well as imaging facilities are particularly vulnerable to acquire the

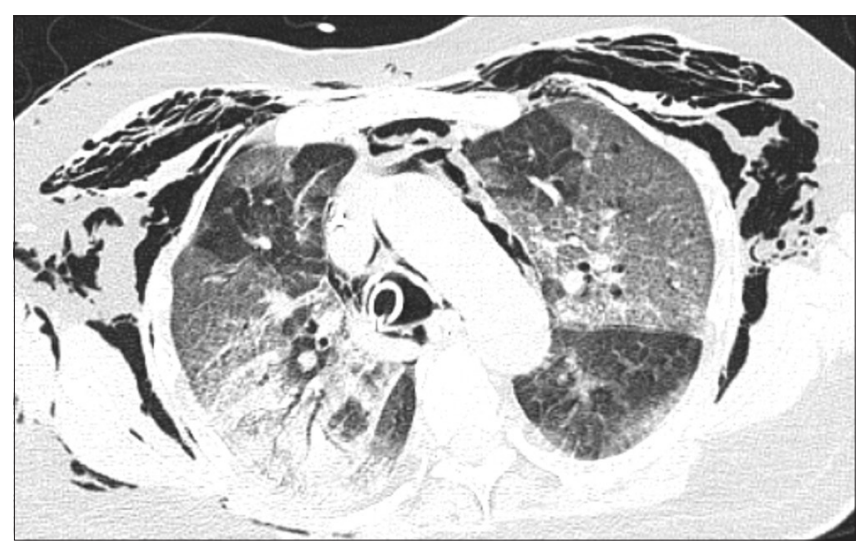

Figure 4: Barotrauma- Extensive ground glass densities and consolidation with superadded mediastinal emphysema and subcutaneous emphysema following complication of mechanical ventilation

infection as well as be carriers to transmit the infection. Care on the prescribed lines of hand hygiene as well as cleansing all contact surfaces where droplets may come in contact is mandatory as these droplets may persist resulting in future contamination. All contact surfaces must be thoroughly and regularly cleaned prior and post examination. Any commercially available cleaning disinfecting agent may be used with a damp cloth. Do not pour it directly on surface. These patient contact surfaces include CT tables, CT Gantry, ultrasound probes and X-RAY detectors. In addition, the workplace for imaging specialists is also shared with co specialists, technicians and nursing staff. All shared contact surfaces like mouse, keyboard and monitors should be regularly cleansed. In fact prior to contact between one user and the other user, the contact surfaces should be sterilised. Pens, chargers etc., should not be shared. Additionally common areas such as door handles, railings etc., also are common contact surfaces which should be cleansed on a regular basis. Of course it is ideal is to avoid contact with shared contact surfaces. This is extremely difficult, but worth training one self.

These are very difficult and uncertain times. We need to keep basic concepts of hand hygiene and high degree of awareness of contact surfaces between humans, regular cleansing, disinfection and prudent use, to curb as much of human to human transmission as possible.

I hope as imaging specialists, we can make a difference in tackling this pandemic.

\section{References}

1. Correlation of Chest $C T$ and RT PCR testing in coronavirus disease 2019 (COVID-19) in china. A report of 1014 cases: Radiology 2020 Feb 26 (Ahead of print). 
2. 2019 coronavirus: The korean clusters- Reuters Graphics- graphics. reuters.com.

3. ACR recommendations on first line usage of Chest radiography and CT Scan for suspected COVID-19 infection: March 11 2020: ACR.org.
This is an open access journal, and articles are distributed under the terms of the Creative Commons Attribution-NonCommercial-ShareAlike 4.0 License, which allows others to remix, tweak, and build upon the work non-commercially, as long as appropriate credit is given and the new creations are licensed under the identical terms.

\begin{tabular}{|l|l|}
\hline \multicolumn{2}{|c|}{ Access this article online } \\
\hline Quick Response Code: & \\
\hline & Website: \\
\hline & www.ijri.org \\
& Dol: \\
\hline
\end{tabular}

Cite this article as: Kohli $\mathrm{A}$. Can imaging impact the coronavirus pandemic? Indian J Radiol Imaging 2020;30:1-3.

Received: 18-Mar-2020

Accepted: 18-Mar-2020

Revision: 18-Mar-2020

Published: 30-Mar-2020 